\title{
Estenosis bronquial distal a la anastomosis en un paciente con trasplante pulmonar bilateral
}

\author{
Bronchial stenosis distal to the anastomosis in a \\ patient with bilateral pulmonary transplant
}

Liliana Fernández T., MD. (1); Luz Fernanda Sua V., MD., PhD. ${ }^{(2)}$; William Martínez, MD. ${ }^{(3)}$; Fernando Sanabria, MD. ${ }^{(3)}$; Carlos García, MD. ${ }^{(4)}$; Mauricio Velásquez, MD. ${ }^{(5)}$

\begin{abstract}
${ }^{(1)}$ Medicina Interna, Neumología y Neumología intervencionista. Línea de Investigación Biomédica en Tórax. Fundación Valle del Lili. Profesora Clínica Asociada, Facultad de Ciencias de la Salud, Universidad Icesi. Cali, Colombia.

(2)Anatomía Patológica y Patología Clínica, Departamento de Patología y Medicina de Laboratorio, Patología Pulmonar, Ciencias Biomédicas. Línea de Investigación Biomédica en Tórax. Fundación Valle del Lili. Facultad de Ciencias de la Salud, Universidad Icesi. Cali, Colombia.

${ }^{(3)}$ Medicina Interna, Neumología. Línea de Investigación Biomédica en Tórax. Fundación Valle del Lili. Facultad de Ciencias de la Salud, Universidad Icesi. Cali, Colombia. ${ }^{(4)}$ Departamento de Imágnes Diagnósticas. Línea de Investigación Biomédica en Tórax. Fundación Valle del Lili. Facultad de Ciencias de la Salud, Universidad Icesi. Cali, Colombia.

${ }^{(5)}$ Cirugía de Tórax. Línea de Investigación Biomédica en Tórax. Fundación Valle del Lili. Facultad de Ciencias de la Salud, Universidad Icesi. Cali, Colombia.

Correspondencia: Liliana Fernández T., correos electrónicos: 1fernandez@fcvl.org, lilianafernandeztrujillo@gmail.com Recibido: 01/02/16. Aceptado: 15/02/16.
\end{abstract}

\section{Introducción}

El trasplante pulmonar es el tratamiento más efectivo para la enfermedad pulmonar en estado terminal. La sobrevida a largo plazo está determinada por la aparición de rechazo crónico (1); la sobrevida a corto plazo, sin embargo, se ve afectada por complicaciones como las relacionadas con la vía aérea (2), la cuales se presentan en el $2 \%$ de los casos, incrementando tres veces el riesgo de muerte y reduciendo en un promedio de $30 \%$ la sobrevida acumulada (3). La complicación de la vía aérea más severa es la dehiscencia de la sutura (4) y la más común es la estenosis, que ocurre usualmente en los primeros seis meses postrasplante y en el $95 \%$ de los casos compromete la anastomosis.

En esta oportunidad se describe el caso de un paciente con estenosis no anastomótica ubicada en el bronquio intermediario derecho distal a la anastomosis (5). En general tiene causas múltiples pero la más importante tiene que ver con la disrupción de la circulación, que ocurre después del trasplante que produce isquemia y lesión hipóxica (3). El manejo involucra dilataciones repetidas y colocación de stents (2).

\section{Caso}

Se trata de un paciente hombre de 29 años con antecedente de fibrosis quística variante doble heterocigoto con múltiples complicaciones, falla respiratoria crónica, infecciones a repetición, colonización por gérmenes resistentes y hospitalizaciones frecuentes, por lo cual se llevó a cabo un protocolo pretrasplante y se realizó trasplante bilateral de pulmón en la Clínica Cardiovascular Santa María de Medellín sin complicaciones, con tiempo de isquemia de pulmón izquierdo 5 horas y derecho 8 horas. Después del procedimiento desarrolló neumonía en el primer mes. Continuó control postrasplante en Cali. Empezó a presentar tos seca y disnea leve, con disminución de los ruidos respiratorios en la base derecha y cambios restrictivos moderados en la espirometría. Se encontró elevación del diafragma derecho en la radiografía de tórax y en la escanografía con reconstrucción 3D se identificó estenosis 
en el bronquio intermediario derecho (BID) (figura 1). En la broncoscopia se observó anastomosis derecha e izquierda sin alteraciones y estenosis severa puntiforme en el BID (figura 2). La biopsia transbronquial de seguimiento postrasplante realizada en ese momento, no mostró algún grado de rechazo (figura 3). El manejo en estos casos involucra broncoplastias con balón repetidas con el fin de aplazar o evitar en lo posible la colocación de stent en la vía aérea comprometida (figura 4).
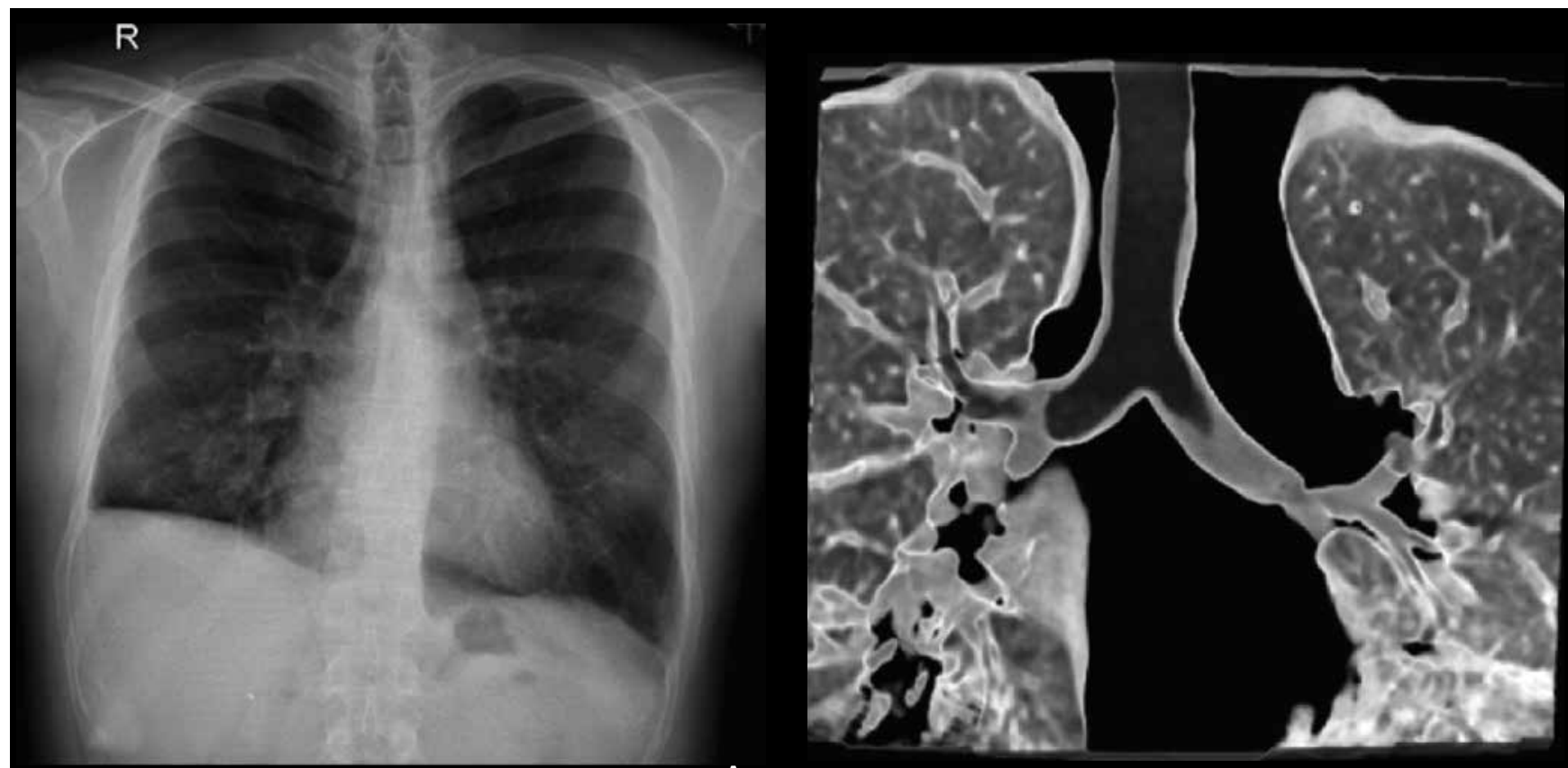

A.
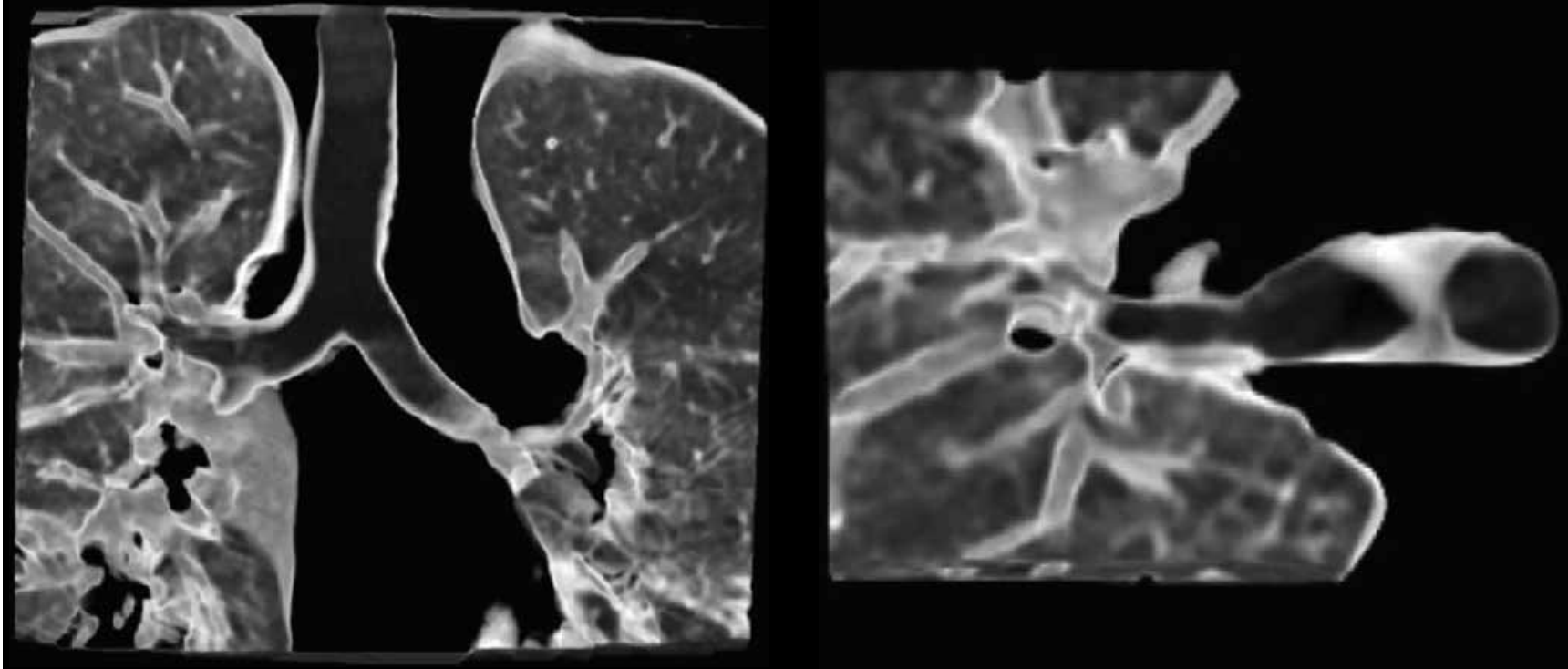

C.

D.

Figura 1. A. Radiografía de tórax con elevación del diafragma derecho sin infiltrados anormales. B, C, D. Tomografía de de tórax con reconstrucción en 3D en la que se aprecia estenosis en el bronquio intermediario derecho. 


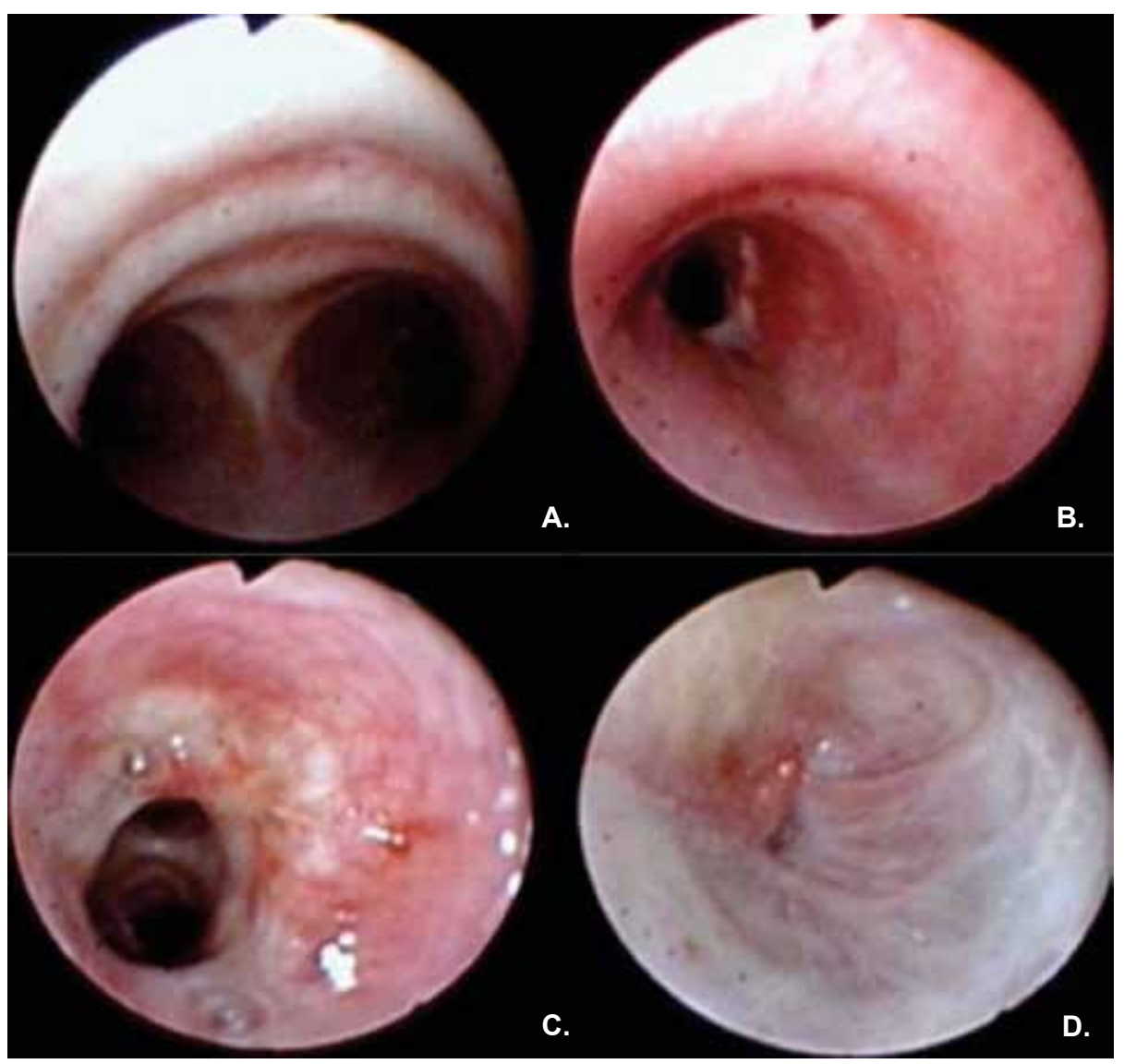

Figura 2. A. Carina principal. B. Anastomosis izquierda normal. C. Anastomosis derecha normal. D. Estenosis del bronquio intermediario distal a la anastomosis.

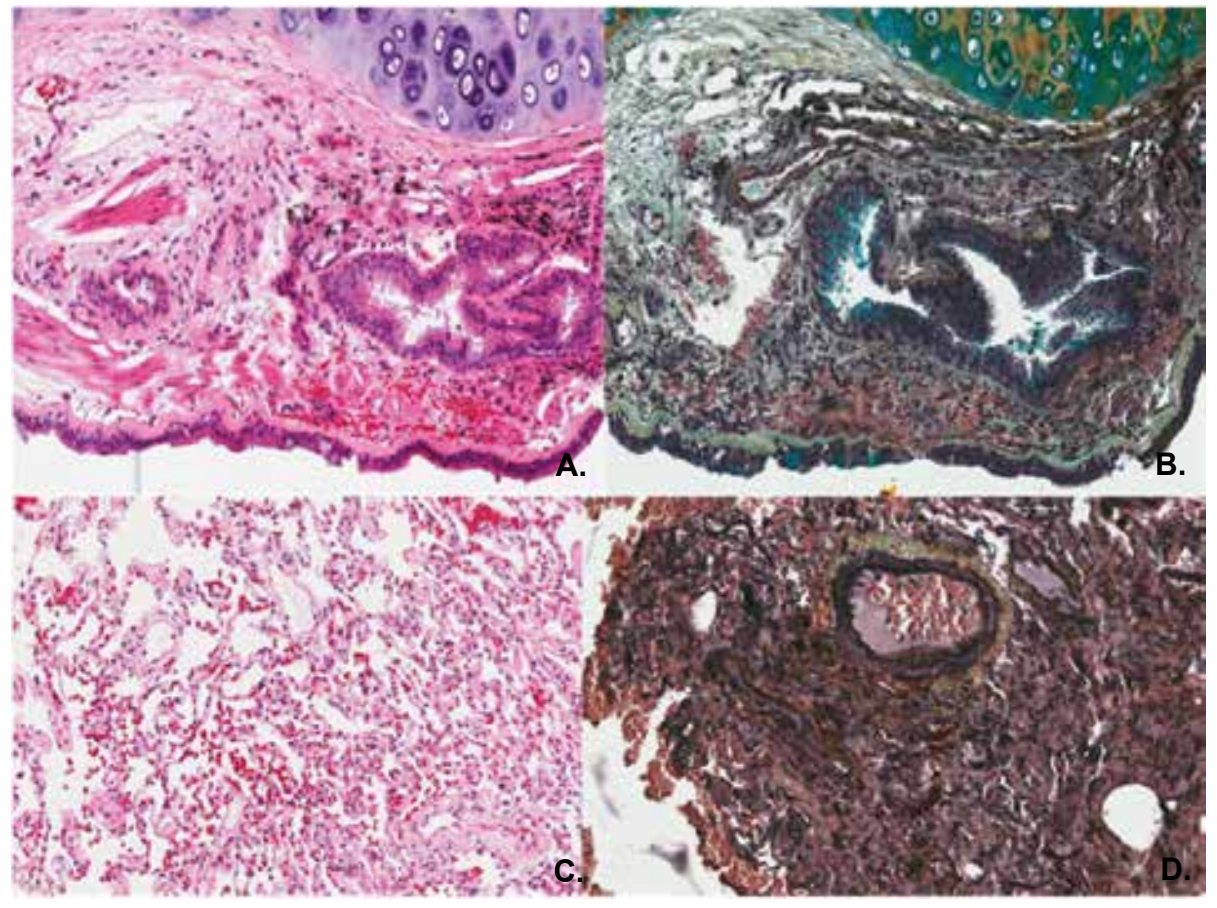

Figura 3. A, B. Pared bronquial representada sin evidencia de rechazo agudo H\&E y Movat. C y D. Parénquima pulmonar sin rechazo agudo (AOB0) H\&E y Movat. 


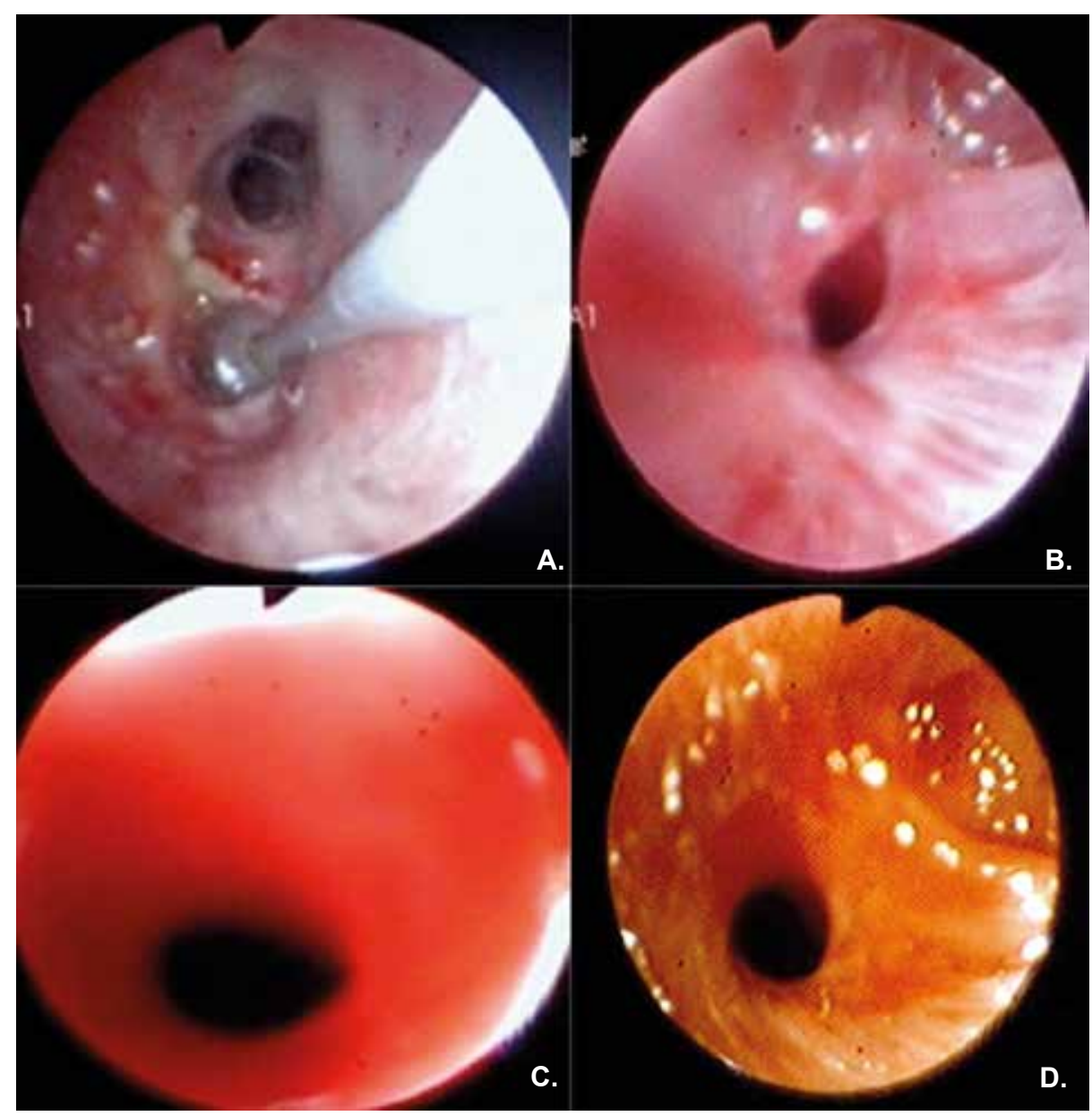

Figura 4. Aspecto del manejo; broncoplastia con balón en la que se logra dilatación progresiva del bronquio intermediario derecho.

\section{Conclusiones}

La estenosis bronquial distal a la anastomosis es una complicación poco frecuente que ocurre después de un trasplante pulmonar. Se debe evaluar por separado respecto a las estenosis más frecuente ubicadas sobre la anastomosis. En la literatura se reportan pocos casos de esta condición (2). En el caso expuesto la dilatación con balón ha dado resultados positivos y ha conducido a la mejoría de los síntomas, la clase funcional y los datos espirométricos.

\section{Conflictos de interés}

Los autores declaran no tener conflictos de interés.

\section{Bibliografía}

1. Weigt SS, DerHovanessian A, Wallace WD, Lynch JP 3rd, Belperio JA. Bronchiolitis obliterans syndrome: the Achilles'heel of lung transplantation. Semin Respir Crit Care Med. 2013;34(3):336-51.

2. Santacruz JF, Mehta AC. Airway complications and management after lung transplantation: ischemia, deshiscence and stenosis. Proc Am Thorac Soc. 2009;6(1):79-93.

3. Awori Hayanga JW, Aboagye JK, Shigemura N, et al. Airway complications after lung transplantation: Contemporary survival and outcomes. J Heart Lung Transplant. 2016;pii:S1053-2498(16)30123-1. doi: 10.1016/j.healun.2016.04.019 [Epub ahead of print]

4. Hayes D, Hatton KW, Feola DJ, Murphy BS, Mullett TW. Airway dehiscence after lung trasplantation in a patient with cystic fibrosis. Respir Care. 2010;55(12):1746-50.

5. Hasegawa T, Iacono AT, Orons PD, et al. Segmental nonanastomotic bronchial stenosis after lung transplantation. Ann Thorac Surg. 2000;69:1020-4. 